\title{
Are Individual Differences in Performance on Perceptual and Cognitive Optimization Problems Determined by General Intelligence?
}

\author{
Nicholas R. Burns, Michael D. Lee, and Douglas Vickers*
}

\begin{abstract}
Studies of human problem solving have traditionally used deterministic tasks that require the execution of a systematic series of steps to reach a rational and optimal solution. Most real-world problems, however, are characterized by uncertainty, the need to consider an enormous number of variables and possible courses of action at each stage in solving the problem, and the need to optimize the solution subject to multiple interacting constraints. There are reliable individual differences in people's abilities to solve such realistic problems. It also seems likely that people's ability to solve these difficult problems reflects, or depends on, their intelligence. We report on a study of $N=101$ adults who completed a series of visual optimization problems (Traveling Salesperson, Minimum Spanning Tree, and Generalized Steiner Tree Problems), as well as a cognitive optimization problem (a version of the Secretary Problem). We also characterized these individuals along three relevant and important cognitive abilities dimensions-fluid ability, visuo-spatial ability, and cognitive processing speed. Modeling of covariance structures indicated that performance on both types of optimization problems relies on general intelligence and raises the possibility that they can be used to assess intelligence.
\end{abstract}

\section{Individual Differences in Problem Solving and Intelligence}

This paper describes a study on individual differences in two classes of decision-making problems: (a) difficult optimization problems that are presented visually and can be thought of as perceptual problems, and (b) a difficult sequential decision-making task that involves uncertainty and places demands on working memory and can be thought of as a cognitive problem.

Recent research has explored whether performance on perceptual optimization problems, particularly the Traveling Salesperson Problem (TSP; Lawler, Lenstra, Rinooy Kan, \& Schmoys, 1985), depends on psychometric intelligence (e.g., Vickers, Butavicius, Lee, \& Medvedev, 2001; Vickers, Mayo, Heitmann, Lee, \& Hughes, 2004; Vickers et al.,

*Department of Psychology, University of Adelaide, Australia

Correspondence concerning this article should be addressed to: Nicholas Burns, School of Psychology, University of Adelaide, South Australia, 5005, Email: nicholas.burns@adelaide.edu.au

The Journal of Problem Solving • volume 1, no. 1 (Fall 2006) 
2004) reported that, in two separate studies, performance on TSP correlated with scores on Raven's Advanced Progressive Matrices (Raven, Court, \& Raven, 1988) to the extent of about $25 \%$ shared variance. Correlations were of similar magnitude for two other perceptual optimization problems known as the Minimum Spanning Tree Problem (MSTP: Ahuja, Magnanti, \& Orlin, 1993) and Generalized Steiner Tree Problem (GSTP: Hwang, Richards, \& Winter, 1992). There are no published reports of relationships between sequential decision-making tasks and psychometric intelligence, but clear and reliable individual differences in performance on one such task, the full-information version of the Secretary Problem, have been described (Lee, O'Connor, \& Welsh, 2004).

Meanwhile, theories on the structure of human intelligence have converged in a model that incorporates at least two strata but arguably three. The first stratum comprises over 60 abilities that are rather narrow and correspond conceptually to Thurstone's Primary Mental Abilities (Thurstone, 1938, 1947). The pattern of intercorrelations among these first-stratum abilities defines about ten broad abilities at the second stratum. These second-stratum abilities include fluid and crystallized intelligence, as first described by Cattell $(1943,1963)$, along with others more recently described, primarily by Horn and co-workers (see Horn \& Noll, 1997). The interpretation of the pattern of intercorrelations among these broad second-order abilities remains controversial. Many researchers and theorists argue for the existence and primacy of a third-stratum general factor ( $g$; see Carroll, 1993; Jensen, 1998), whereas others dismiss the importance of $g$ (e.g., Stankov, 2002). Deary and Caryl (1997) make the point that whether the broad second-order abilities or the putative third-order general factor is considered most important depends on the particular research question at hand. The point of all this, however, is that it is no longer sufficient to attempt to understand human intelligence, its determinants, or consequences, in terms of scores on a single psychometric instrument such as Raven's Matrices.

Here, we examine the relationship of individual differences on difficult perceptual and cognitive problems with psychometric tests chosen to define the three most relevant broad cognitive abilities identified by modern theories of human intelligence: fluid ability (Gf), visuo-spatial ability (Gv), and cognitive processing speed (Gs).

\section{Perceptual Optimization Problems}

As described in more detail elsewhere (Vickers et al., 2004), much research into the relationship between problem solving processes and other cognitive abilities has concentrated either on knowledge-lean problems suitable for experimental study or on complex but domain-specific real-world problems. Vickers et al. argued that difficult combinatorial optimization problems have the advantage that, although there is often no algorithm that can produce a definitive solution in a reasonable time, they can be simply stated and readily understood. Moreover, they can be representative of 
real-world situations, and human performance on them is often close to optimal (e.g., Graham, Joshi, \& Pizlo, 2000).

In the TSP, participants are given a set of $n$ interconnected nodes that lie on a 2-D Euclidean space, and they must devise an itinerary that visits each node exactly once, returns to the starting node, and ensures that the total length of the tour is as short as possible. To arrive at a definitive solution to this problem entails an exhaustive consideration of ( $n-1) ! / 2$ pathways (see Figure 1a).

In MSTP, participants are required to find the shortest path that directly links all the nodes in an array. This problem has fewer constraints than TSP because the path does not have to be continuous and closed and a node can be connected by more than two links. The optimal solution is an open, branching path system that directly links all the nodes and in which branches occur at the nodes (see Figure $1 \mathrm{~b}$ ).

In the elementary three-node Steiner Tree Problem, the shortest path connecting three nodes in a plane can be found by determining the so-called Fermat point and creating a path of links that branch at that point (as illustrated in Figure 1c). GSTPs contain more than three nodes, and the solutions to these problems look like combinations of solutions found in three-node Steiner Tree problems with additional nodes (P1, P2, . . $\mathrm{Pk}$ ) as branch points to create the minimum connections between the original nodes, as illustrated in Figure 1d.

\section{Figure 1.}

(a) Fifty-node TSP array; (b) fifty-node MTSP array. For both (a) and (b) the optimal solution is shown as solid connecting edges. (c) The Fermat point (P) for a simple Steiner Tree problem as an open circle, with the optimal solution shown by broken lines. (d) Fifteen-node GSTP with the interpolated open points (open circles) and the optimal tree solution (broken lines) found by a computer algorithm.

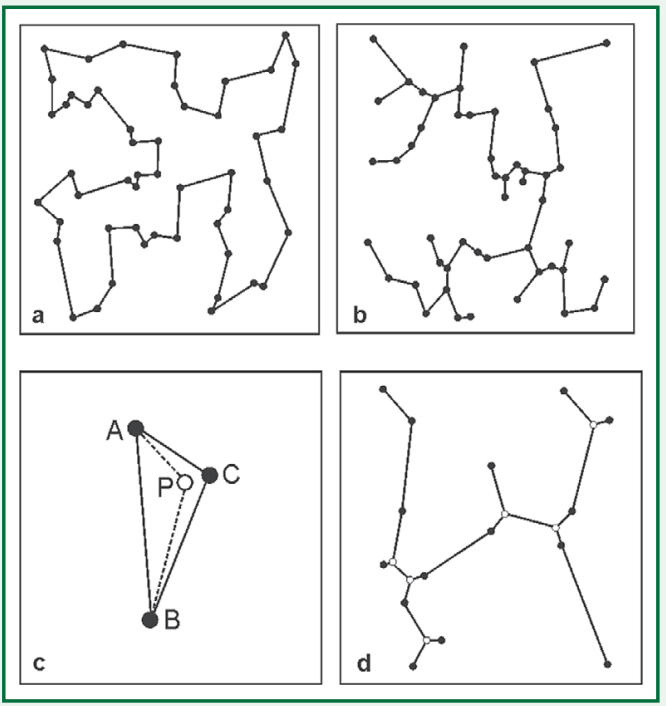


Vickers et al. (2004) showed that performance on versions of these three tasks was characteristic of an individual, with the average intercorrelation among the three tasks implying about $50 \%$ shared variance. Moreover, correlations on performance across two rotated versions of these tasks were good, with the mean across three tasks being about $r=.7$.

\section{Cognitive Optimization Problem}

Many real-world decision-making problems are sequential. A series of choices is made available over time, and it is often efficient (and sometimes even necessary) to make a selection without waiting to be presented with all of the alternatives. This decisionmaking scenario has the same essential features as a recreational mathematics problem known as the Secretary Problem (see Ferguson, 1989, for a historical overview). In Secretary Problems, participants are presented with a sequence of possible choices and must decide whether to accept or reject each possibility in turn. The so-called full information version of the problem presents participants with a score from a known distribution for each possibility; the goal is to choose the maximum score in the sequence, and any incorrect decision is equally wrong. Gilbert and Mosteller (1966) showed that the optimal decision rule for these problems requires choosing the first value that exceeds a threshold level for its position in the sequence.

Because they are not inherently perceptual, Secretary Problems allow consideration of whether results obtained with perceptual optimization problems generalize to cognitively based problem solving. Secretary Problems also introduce uncertainty and place demands on working memory. While visual problems like TSPs are combinatorially large, the basic information about distances between points is always perceptually available in a complete and certain form to the problem solver. By contrast, the sequences of information in secretary problems are stochastic and presented only temporarily, requiring people to deal with uncertainty and to rely on their memory.

\section{The Current Study}

To understand better the previously reported relationship between performance on difficult optimization problems and one, albeit limited, measure of intelligence and to extend that line of research to include a sequential decision-making problem, we administered TSPs, MSTPs, GSTPs, and Secretary Problems as well as marker tests for Gf, Gv, and Gs. We used a sample of sufficient size to model relationships of the problem-solving tasks and psychometric tasks using analysis of covariance structures. A series of alternative theoretically plausible models were compared to delineate further the relationships reported by Vickers et al $(2001,2004)$. 


\section{Methods}

\section{Participants}

There were $N=101$ participants (58 males; mean age $=25.3, S D=7.6$ years) recruited from the general community via advertisements and word-of-mouth. All were paid A $\$ 30$ at completion of the study. All participants affirmed informed consent in writing after reading an information sheet on the study, which was approved by the University of Adelaide Human Research Ethics Committee.

\section{Materials}

\section{Perceptual Optimization Problems}

Problems were presented one at a time in a $15 \mathrm{~cm} \times 15 \mathrm{~cm}$ square in the center of a standard 19-inch computer display. Participants could begin at any point by left-clicking on a node (or on a location where they wished to establish a new node) with the computer mouse. They drew a path by dragging the mouse cursor to a subsequent node and releasing the button, causing a straight line to be drawn between that node and the previously visited node. By right-clicking on a link (or created node) to select it and then pressing the delete key on the keyboard, participants could undo any links or nodes they had drawn. Participants were thus free to connect the nodes in any order, to work alternately from two nodes, or to work on several separated clusters of nodes. If a participant's completed solution was invalid (e.g., because not all TSP nodes had been connected), a warning message was posted on the screen and the participant was obliged to construct a valid solution before proceeding to the next problem.

Participants completed one 30-, 60-, and 90-node TSP; the same for MSTP; and one 15-, 20-, and 25-node GSTP. Participants were given verbal instructions, and there were also instructions on the computer screen that could be redisplayed at any time by clicking on a button. There were no time constraints on performance, but participants were asked to complete the tests as quickly and as accurately as they could.

For TSP, participants were asked to connect all the nodes in a continuous tour that visited each node once only, returned to the starting node, and was as short as possible. In the case of MSTP, participants were asked to connect all the nodes by making a system of paths between them, using as many paths as necessary, but only between existing nodes, and creating a path system with the shortest possible overall length. For GSTP, participants were asked to connect all the nodes by making a system of paths with the shortest overall length. In this case, however, participants were told that the existing nodes did not have to be linked directly. If they wished, participants could create additional nodes, and they were required to create at least one additional node. 


\section{Secretary Problem}

Each participant completed two sets of problems presented on a computer. The first set consisted of 40 problems of length 5, and the second 40 problems of length 10 . Participants did the sets in the same order-length 5, then length 10-but the order of the problems within each set was randomized across participants. For each problem, the participants were told that the values they would see were dollar amounts ranging from 0.00 to 100.00 . They were told the length of the sequence and were instructed to choose the maximum value. It was emphasized that (a) the values were uniformly and randomly distributed between 0.00 and 100.00 , (b) a value could only be chosen at the time it was presented, (c) the goal was to select the maximum value with any selection below the maximum being completely incorrect, and ( $d$ ) if no choice had been made when the last value was presented, they would be forced to choose this value. As each value was presented, its position in the sequence was shown, together with "yes" and "no" response buttons.

\section{Psychometric Tests}

Participants completed custom computerized adaptations of the Raven Standard Progressive Matrices (SPM; Raven, 1956), Cattell Culture Fair Test Scale 2 Form A (CCF; Cattell \& Cattell, 1959), and Spatial Relations from the Woodcock-Johnson Psycho-Educational Battery-Revised (WJ-R; Woodcock \& Johnson, 1989). For SPM, participants worked through a series of up to 60 matrices questions; we used a timed version with a 20-minute limit. The CCF has four sections: Series, Classification, Matrices and Conditions. Participants worked through examples followed by the 12,14, 12, and 8 items for each section, respectively. Each section had a fixed time limit, and the total test lasted 12.5 minutes. Spatial Relations presented participants with a large shape comprised of smaller components. Next to this was displayed a set of small shapes, and participants decided which of these together constituted the large shape. The items became progressively more difficult with the shapes becoming more complex and with more components in each large shape. The test comprised 33 items and finished when the participants answered incorrectly on six items in a row or when they had completed all items. A fourth computerized test was Picture Swaps, based on a test described by Crawford (1988). Participants are required mentally to swap the order of three pictures presented on the computer screen according to instructions also presented on the screen and then to indicate the final order of the pictures. Once they had worked out the answer, they pressed the space bar on the keyboard to bring up another screen displaying the six possible solutions numbered 1 to 6 . Participants responded via the keyboard number pad. There were four difficulty levels for the test, which differed in the number of swaps required (one, two, three, or four swaps). There were 6 items for both the one- and twoswap levels and 12 items for the three- and four-swap levels. 
There were also three paper-and-pencil tests, Space Relations from the Differential Aptitude Tests Form W (Bennet, Seashore, \& Wesman, 1989), Digit Symbol from the Wechsler Adult Intelligence Scale-III (Wechsler, 1997), and Visual Matching from the WJ-R (Woodcock \& Johnson, 1989). Apart from using only the 30 odd-numbered items from Space Relations, these tests were administered according to instructions in the respective manuals. Space Relations had a time limit of 12.5 minutes; the other two tests had time limits of 3 minutes.

\section{Procedure}

Participants attended the laboratory either individually or two at a time. Paper-andpencil tests were completed first in the order described above, followed by TSP, MSTP, GSTP, and Secretary Problem. The computerized psychometric tests were then completed in the order described above. There were two workstations separated by a partition so that participants attending in pairs were isolated from each other when completing the computerized tests. Testing sessions lasted about two hours.

\section{Results}

Participants' solution lengths for each perceptual optimization problem were expressed as a proportion above the benchmark (PAB), or best known solution length, for that problem. Thus, a PAB score for an optimal solution would be 0 , and any solution that exceeded the benchmark would have a positive score. The final measures used for TSP, MSTP, and GSTP in subsequent analyses were the mean PABs for the three problems of each type. For Secretary Problems, the measure used was the mean proportion of trials for which the participant chose in accordance with the prediction of the optimal decision rule, averaged across length- 5 and length-10 problems.

For SPM, it is known that some items measure visuo-spatial ability whereas others measure verbal-analytical reasoning (Lynn, Allik, \& Irwing, 2004). We therefore created item parcels by summing across these different types of items. Hereafter, we designate these as SPM-VSp and SPM-VAR, respectively. A similar situation applies to CCF (see Burns \& Nettelbeck, 2003). Again, we created item parcels hereafter designated CCF-VSp and CCF-VAR. For Spatial Relations, Picture Swaps, Space Relations, Digit Symbol, and Visual Matching, total items correct scores were used. Table 1 shows descriptive statistics for measures used in subsequent analyses. SPM-VSp, CCF-VSp, Spatial Relations, and Space Relations were included as measures of Gv; SPM-VAR, CCF-VAR, and Picture Swaps as measures of Gf; and Digit Symbol and Visual Matching as measures of Gs. 


\section{Table 1}

Descriptive Statistics and Pearson Correlations for Optimization Problems, Secretary Problem, and Psychometric Measures for $\mathrm{N}=101$ Participants.

\begin{tabular}{|l|l|c|c|c|c|c|c|c|c|c|c|c|c|c|}
\hline $\mathbf{1}$ & \multicolumn{1}{|c|}{ TSP } & $\mathbf{1}$ & $\mathbf{2}$ & $\mathbf{3}$ & $\mathbf{4}$ & $\mathbf{5}$ & $\mathbf{6}$ & $\mathbf{7}$ & $\mathbf{8}$ & $\mathbf{9}$ & $\mathbf{1 0}$ & $\mathbf{1 1}$ & $\mathbf{1 2}$ & $\mathbf{1 3}$ \\
\hline 2 & MSTP & .54 & & & & & & & & & & & & \\
\hline 3 & GSTP & .69 & .78 & & & & & & & & & & & \\
\hline 4 & SecProb & -.14 & -.10 & -.13 & & & & & & & & & & \\
\hline 5 & SPM-VSp & -.27 & -.22 & -.39 & .21 & & & & & & & & & \\
\hline 6 & SPM-VAR & -.36 & -.29 & -.43 & .23 & .52 & & & & & & & & \\
\hline 7 & CCF-VSp & -.25 & -.20 & -.23 & .14 & .40 & .38 & & & & & & & \\
\hline 8 & CCF-VAR & -.31 & -.20 & -.31 & .24 & .28 & .42 & .48 & & & & & & \\
\hline 9 & SpatRel & -.36 & -.27 & -.35 & .25 & .45 & .49 & .50 & .32 & & & & & \\
\hline 10 & PicSwaps & -.25 & -.19 & -.28 & .37 & .58 & .35 & .39 & .31 & .46 & & & & \\
\hline 11 & SpaceRel &.- .38 & -.11 & -.29 & .20 & .30 & .32 & .47 & .51 & .35 & .42 & & & \\
\hline 12 & DigSym & -.09 & -.12 & -.10 & .15 & .26 & .33 & .13 & .32 & .08 & .34 & .19 & & \\
\hline 13 & VisMat & -.14 & -.25 & -.14 & .19 & .34 & .33 & .23 & .28 & .15 & .37 & .22 & .63 & \\
\hline & Mean & .093 & .061 & .140 & .711 & 19.1 & 6.98 & 20.4 & 6.50 & 20.5 & 28.4 & 25.9 & 85.5 & 42.5 \\
\hline & SD & .041 & .073 & .104 & .126 & 2.31 & 3.75 & 3.26 & 2.22 & 4.63 & 7.62 & 4.84 & 15.4 & 5.54 \\
\hline
\end{tabular}

Note: TSP is Traveling Salesperson Problem; MSTP is Minimum Spanning Tree Problem; GSTP is Generalized Steiner Tree Problem; SecProb is Secretary Problem; SPM-VSp is Standard Progressive Matrices-Visuo-Spatial; SPM-VAR is Standard Progressive Matrices-Verbal Analytic Reasoning; CCFVSP is Cattell Culture Fair-Visuo-Spatial; CCF-VAR is Cattell Culture Fair-Verbal Analytic Reasoning; SpatRel is Spatial Relations; PicSwaps is Picture Swaps; SpaceRel is Space Relations; DigSym is Digit Symbol; VisMat is Visual Matching.

The first set of models to be compared here is as follows: first, a model where all variables load a single latent variable; thus, this model proposes that covariances of all variables depend on a single construct, general intelligence $(g)$. This is referred to hereafter as Model 1. Second, a model is proposed where performance on the problemsolving tasks used here depends on a "problem solving ability" different from but related to those currently described in psychometric theories of intelligence. This is referred to as Model 2. The next model allows that the combinatorial optimization problems depend on Gv, whereas Secretary Problem depends on Gf. This model, hereafter Model 3, arises prima facie on the basis of the requirements of these classes of tasks, as described above. All models were fitted using AMOS 5 (Arbuckle, 2003) or LISREL 8.5 (Jöreskog \& Sörbom, 2003).

Comparison of these types of models, often referred to generically as structural equation models, should be done using a range of fit criteria; specifically, goodness-offit and model complexity should be considered (Kline, 2005). Here we use the Bayesian Information Criterion (BIC; Schwartz, 1978; Raftery, 1995), the Root Mean Square Error of 
Approximation (RMSEA; Steiger \& Lind, 1980) along with its 90\% confidence interval, and the model chi-square. The BIC takes account of sample size and penalizes model complexity; comparison of BIC across models gives an indication of which is the better model taking account of the parsimony principle - the lower the BIC, the better the model; the RMSEA is a parsimony-adjusted index where a commonly adopted rule-of-thumb is that values less than about .05 indicate close approximate fit and RMSEA greater than or equal to .10 suggests poor fit (see e.g., Browne \& Cudeck, 1993). The model chi-square tests the hypothesis that the model is correct but is sensitive to the size of correlations and to sample size. Commonly, to overcome these problems, it is divided by the model degrees of freedom (normed chi-square), and a rule of thumb is that this value should be less than about two for a well fitting model (see Bollen, 1989). Table 2 shows the fit criteria for the first set of models considered.

\section{Table 2}

Fit Criteria for Three Models Showing Bayesian Information Criterion (BIC), Root Mean Square Error of Approximation (RMSEA) and Its $90 \% \mathrm{Cl}$, and Likelihood Ratio Chi-Square. Forcomparison purposes, the relevantstatistics are shown for the saturated model, where no constraints are imposed and fit is perfect, and for the null model where population correlations are assumed to be zero.

\begin{tabular}{|l|c|c|c|c|}
\hline \multicolumn{1}{|c|}{ Model } & BIC & RMSEA Cl $_{\mathbf{9 0}}$ & $\chi^{\mathbf{2}}$ (df) & $\boldsymbol{p}$ \\
\hline 1 & 356.5 & $.16[.14, .18]$ & $236.5(65)$ & $<.001$ \\
\hline 2 & 239.5 & $.07[.04, .10]$ & $91.8(59)$ & .004 \\
\hline 3 & 314.1 & $.14[.11, .16]$ & $180.3(62)$ & $<.001$ \\
\hline Saturated & 420.0 & - & $0(0)$ & - \\
\hline Null & 596.6 & $.24[.22, .26]$ & $536.6(78)$ & $<.001$ \\
\hline
\end{tabular}

Note: For comparison purposes, the relevant statistics are shown for the saturated model, where no constraints are imposed and fit is perfect, and for the null model, where population correlations are assumed to be zero.

None of the models fit the data well. Examination of the parameters of the bestfitting model, that is, Model 2, showed that GSTP had an estimated standardized regression weight on the latent variable representing problem solving of .98, whereas TSP, MSTP, and Secretary Problem had weights of .70, .78, and .13, respectively. These estimates suggest misspecification of this model arising due to collinearity of the perceptual optimization problems. Certainly, the zero-order correlation between GSTP and MSTP is .78, $\mathrm{Cl}_{95}=[.69, .85]$. Furthermore, using these two variables to predict TSP by linear regression resulted in a model with multiple $R=.69$ but where the variance inflation factor for both GTSP and MSTP was 2.47. Therefore, these three variables were combined by standardizing each and taking the mean. This new measure is hereafter referred to as 
Perceptual Optimization Problems. This variable was incorporated into a new model, hereafter Model 4 (see Figure 2), based on Model 3. Estimation of this model highlighted that Gf and Gv were perfectly correlated, so to properly estimate the model, the covariances between $\mathrm{Gf}$ and $\mathrm{Gs}$ and between $\mathrm{Gv}$ and $\mathrm{Gs}$ were constrained to be equal. It can be seen in Figure 2 that Model 4 is better than any of the others considered so far. It should also be noted that this model can be equivalently represented with a higherorder general factor instead of correlated first-order factors.

\section{Figure 2.}

Model 4 showing maximum likelihood estimates of standardized regression weights and fit criteria. All estimated loadings and covariances differ from zero $p<.001$. See Tables 1 and 3 for variable names.

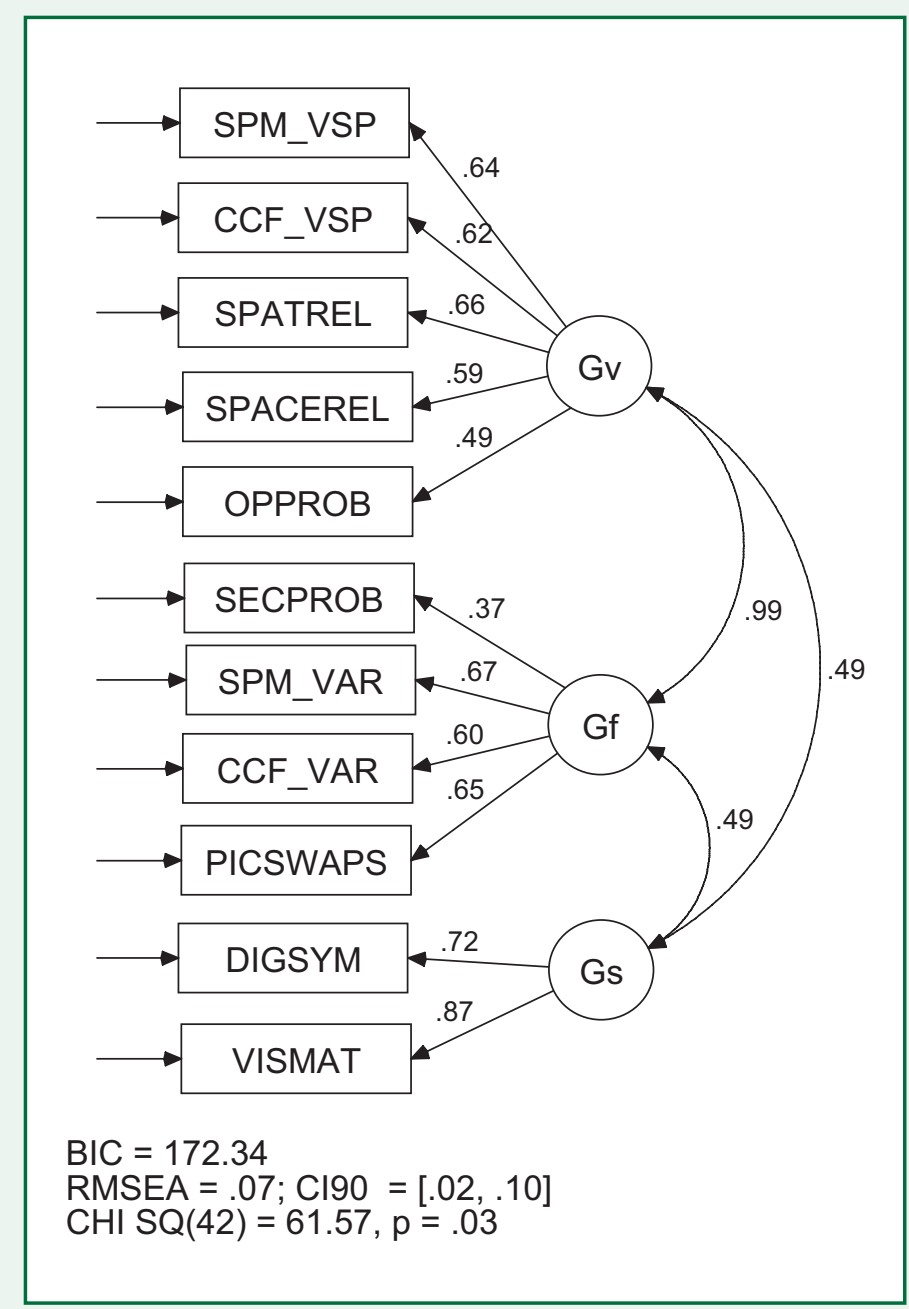

The final model to be considered, hereafter Model 5 (see Figure 3), is suggested by examination of Figure 2 and has the interesting property of allowing all variables to load on a single latent variable representing $g$ while at the same time allowing Digit Symbol and Visual Matching also to load on an orthogonal latent variable representing Gs. 
Model 5 is, thus, a hierarchical model with uncorrelated factors and is presented here because the standardized regression coefficients for Digit Symbol and Visual Matching on $g$ have effectively been partialled of the speed component of these measures, thereby allowing direct comparison of the $g$-loadings of all variables (see Table 3 ). Thus, Model 5 allows that, in this sample and with these variables, Gf and Gv cannot be distinguished from each other (this is a not uncommon outcome; see Danthiir, Roberts, Pallier, \& Stankov, 2001), or from $g$ (see also Gustafsson \& Undheim, 1996). By contrast Gs is well marked. Model 5 has $\mathrm{BIC}=167.7$, $\mathrm{RMSEA}=.07 . \mathrm{Cl}_{90}=[.02, .10]$, and $\chi^{2}(43)=61.6, p=.03$.

\section{Figure 3.}

Conceptual diagram for Model 5, a hierarchical model with uncorrelated factors, which allows for estimation of g-loadings for all variables with Digit Symbol and Visual Matching residualized for their speed component. See Tables 1 and 3 for variable names.

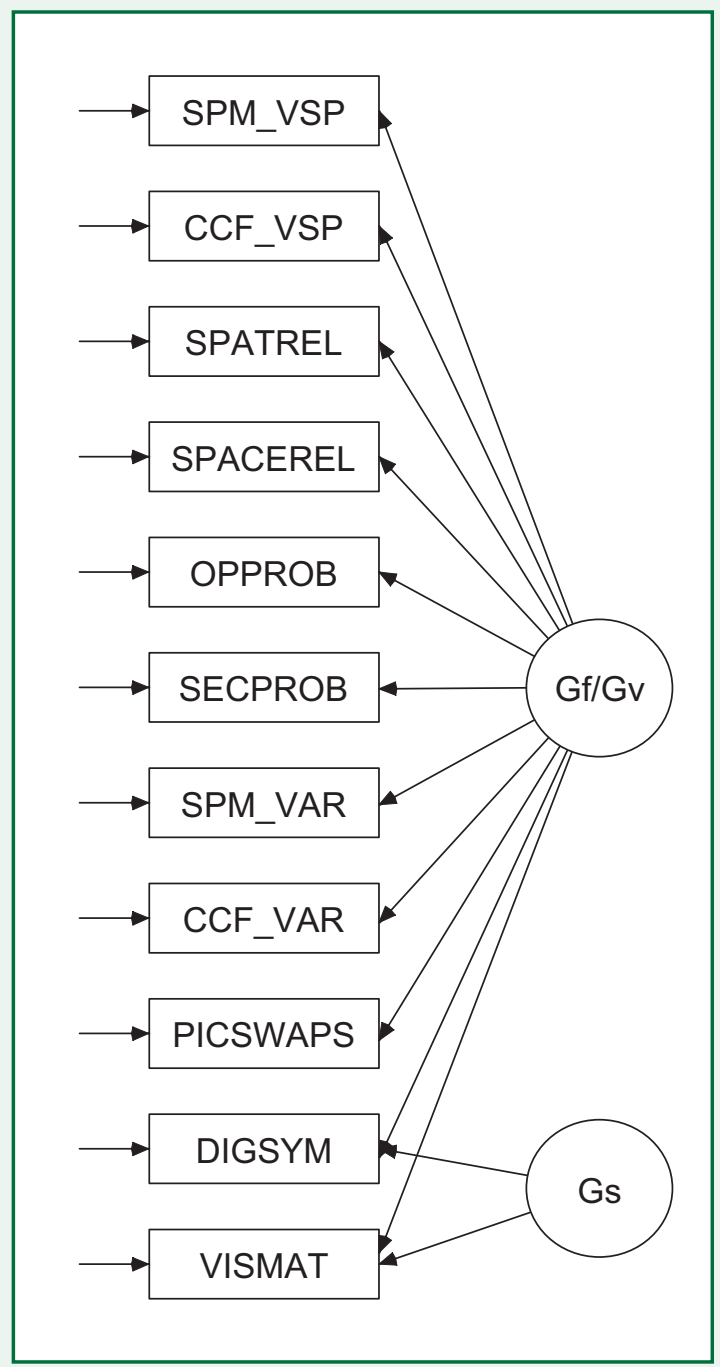




\section{Table 3}

Maximum Likelihood Estimates of Loadings on Two Orthogonal Latent Variables.

\begin{tabular}{|l|c|c|}
\hline & g & Gs \\
\hline OpProb & .492 & - \\
\hline SecProb & .368 & - \\
\hline SPM-VSp & .642 & - \\
\hline SPM-VAR & .668 & - \\
\hline CCF-VSp & .617 & - \\
\hline CCF-VAR & .601 & - \\
\hline SpatRel & .662 & - \\
\hline PicSwaps & .649 & - \\
\hline SpacRel & .594 & .828 \\
\hline DigSym & .357 & .575 \\
\hline VisMat & .433 & - \\
\hline
\end{tabular}

Note: See Table 1 for variable names, except OpProb is mean of standardized scores for TSP, MSTP, and GSTP. All estimated loadings differ from zero $p<.001$. Loadings fixed to zero marked as - .

\section{Discussion}

The main question of interest here concerned the relationship between individual differences in performance on difficult optimization problems and constructs described in modern psychometric theories of human intelligence. The two classes of problemsolving tasks we used had the very desirable characteristic of being representative of real-world decision-making scenarios. Definitions of intelligence invariably make reference to problem solving along with the ability to reason, plan, think abstractly, comprehend complex ideas, learn quickly, and learn from experience (e.g., Neisser et al., 1996), but a common criticism of traditional intelligence testing is that it fails to capture the requirements of real-world problem solving. Thus, one could imagine that, rather than presenting TSP problems in the abstract format used here, they could be embedded within a cover story suitable, for example, for use with children, whereby their face validity as real-world problems becomes evident. A similar argument can be made about the Secretary Problem; for example, Lee et al. (2004) described the Secretary Problem as having characteristics similar to a police suspect identification line-up (albeit with some unlikely constraints placed on the scenario).

When difficult optimization problems were jointly examined with a test of intelligence, robust relationships were found (Vickers et al., 2004). Here, we acknowledged that human intelligence is better described in terms of a hierarchical model of related abilities with constructs being most narrow at the foot of the hierarchy and very general 
at the apex. We sampled abilities with the aim of defining fluid ability (Gf), which includes reasoning abilities and working memory and is arguably indistinguishable from $g$; visuospatial ability (Gv) - that is, abilities in apprehending the forms, shapes, and positions of objects and forming and manipulating mental representations of those forms, shapes, and positions-and cognitive processing speed (Gs), which many view as fundamental to individual differences in intelligence.

We found that both a single general factor model (Model 1) and a model incorporating a separate problem-solving ability (Model 2 ) were inadequate to account for the data we observed. A model (Model 4) that allowed a variable representing the perceptual optimization problems to load Gv and the Secretary Problem to load Gf, with both Gv and Gf correlated with each other and with Gs provided a good account of the data. Not reported here were variations on this model with the optimization and Secretary Problems loading either on the alternate or on both latent variables (i.e., Gv and Gf); these were poorer fitting models. Thus, at this level of analysis, both classes of optimization problems were shown as being relatively good measures of Gv or Gf, respectively.

The final model considered comprised two orthogonal latent variables. All variables loaded on general ability, but the two cognitive speed measures also loaded on Gs. This arrangement allowed us to assess the $g$-loadings of all measures. The main outcome was that the variable representing the combinatorial optimization problems had a loading of about .5 on $g$, whereas Secretary Problem had a loading of about .4. These loadings are high enough to encourage us to speculate that both of these types of problems could prove useful as measures of cognitive ability.

Much work is required before, for example, TSP or Secretary Problem could be routinely used to assess intelligence; clearly, performance on both types of problem should be validated against criteria other than just intelligence tests to determine their predictive validity. This is so, of course, because different aspects of cognitive performance may predict different life outcomes. Tests of Gv, for example, seem to best predict job performance in tradespeople and artisans but are also required by mathematicians and physicists (see McGrew, 2005). Optimization problems may fill a gap in current test batteries because they are demonstrably related to real-world problem solving, and performance on them perhaps does not depend on acculturated knowledge, although this does remain to be empirically tested.

Finally, we suggest that studies on the relationships between problems like TSP and Secretary Problem and intelligence should consider: (a) the developmental trajectories of performance on these problem-solving tasks in comparison to those of other cognitive abilities, and (b) relationships between solution times on problem-solving tasks and performance on so-called elementary cognitive tasks, including psychomotor speed, which correlate substantially with cognitive abilities. Of promise also is the application of models on TSP and Secretary Problem performance to a better understanding of individual differences in human intelligence. 


\section{References}

Ahuja, R. K., Magnanti, T. L., \& Orlin, J. B. (1993). Network flows: Theory, algorithms, and applications. NY: Prentice Hall.

Arbuckle, J. L. (2003). AMOS 5. Chicago, IL: Small Waters.

Bennet, G. K., Seashore, H. G., \& Wesman, A. G. (1989). Differential aptitude tests. Marickville, NSW: Psychological Corporation.

Bollen, K. A. (1989). Structural equations with latent variables. NY: Wiley.

Browne, M. W. \& Cudeck, R. (1993). Alternative ways of assessing model fit. In K. A. Bollen and J. S. Long (Eds.), Testing structural equation models (pp. 445-455). Newbury Park, CA: Sage.

Burns, N. R., \& Nettelbeck, T. (2003). Inspection time in the structure of cognitive abilities: Where does IT fit? Intelligence, 31, 237-255.

Carroll, J. B. (1993). Human cognitive abilities: A survey offactor analytic studies. NY: Cambridge University Press.

Cattell, R. B. (1943). The measurement of adult intelligence. Psychological Bulletin, 40, 153193.

Cattell, R. B. (1963). Theory for fluid and crystallized intelligence: A critical experiment. Journal of Educational Psychology, 54, 1-22.

Cattell, R. B. \& Cattell, A. K. S. (1959). The Cattell Culture Fair Test. Champaign, IL: IPAT.

Crawford, J. D. (1988). Intelligence, task complexity and tests of sustained attention. Unpublished PhD thesis, the University of New South Wales.

Danthiir, V., Roberts, R. D., Pallier, G., \& Stankov, L. (2001). What the nose knows: Olfaction and cognitive abilities. Intelligence, 29, 337-361.

Deary, I. J., \& Caryl, P. G. (1997). Neuroscience and human intelligence differences. Trends in Neurosciences, 20, 365-371.

Ferguson, T. S. (1989). Who solved the Secretary Problem? Statistical Science, 4, 282-296.

Gilbert, J. P., \& Mosteller, F. (1966). Recognizing the maximum of a sequence. Journal of the American Statistical Association, 61, 35-73.

Graham, S. M., Joshi, A., \& Pizlo, Z. (2000). The Traveling Salesman Problem: A hierarchical model. Memory and Cognition, 28, 1191-1204.

Gustafsson, J.-E. \& Undheim, J. O. (1996). Individual differences in cognitive functions. In D. C. Berliner and R. C. Calfee (Eds.), Handbook of educational psychology (pp. 186-242). NY: Macmillan.

Horn, J. L., \& Noll, J. (1997). Human cognitive capabilities: Gf-Gc theory. In D. P. Flanagan, J. L Genshaft, \& P. L. Harrison (Eds.), Contemporary intellectual assessment: Theories, tests and issues, (pp. 53-91). NY: Guilford.

Hwang, F. K., Richards, D. S., \&Winter, P. (1992). The Steiner Tree Problem. Amsterdam: Elsevier. Jensen, A. R. (1998). The $g$ factor: The science of mental ability. NY: Praeger.

Jöreskog, K. G. \& Sörbom, D. (2003). LISREL 8.5. Chicago: Scientific Software International.

Kline, R. B. (2005). Principles and practice of structural equation modeling (2nd ed.). NY: Guilford. Lawler, E. L., Lenstra, J. K., Rinooy Kan, A. H. G., \& Schmoys, D. B. (1985). The Traveling Salesman Problem: A guided tour of combinatorial optimization. Chichester, UK: Wiley. 
Lee, M. D., O'Connor, T. A., \& Welsh, M. B. (2004). Decision-making on the full-information Secretary Problem. In K. Forbus, D. Gentner \& T. Regier (Eds.), Proceedings of the 26th Annual Conference of the Cognitive Science Society, (pp. 819-824). Mahwah, NJ: Erlbaum. Lynn, R., Allik, J., \& Irwing, P. (2004). Sex differences on three factors identified in Raven's Standard Progressive Matrices. Intelligence, 32, 411-424.

McGrew, K. S. (2005). The Cattell-Horn-Carroll theory of cognitive abilities: Past, present, and future. In D. P. Flanagan \& P. L Harrison (Eds.), Contemporary intellectual assessment: Theories, tests, and issues (2nd ed., pp. 136-181). NY: Guilford Press.

Neisser, U., Boodoo, G., Bouchard, T. J., Boykin, A. W., Brody, N., Ceci, S. J, et al. (1996). Intelligence: Knowns and unknowns. American Psychologist, 51, 77-101.

Raftery, A. (1995). Bayesian model selection in social research. In P. Marsden (Ed.), Sociological Methodology, (pp. 111-163). Oxford: Blackwell.

Raven, J. C. (1956). Standard progressive matrices. Hawthorn, Vic: Australian Council for Educational Research.

Raven, J. C., Court, J. H., \& Raven, J. (1988). Manual for Raven's progressive matrices and vocabulary scales. Section 4: Advanced progressive matrices. London: Lewis.

Schwarz, G. (1978). Estimating the dimension of a model. Annals of Statistics, 6, 461-464.

Stankov, L. (2002). g: A diminutive general. In R. J. Sternberg \& E. L. Grigorenko (Eds.), The general factor of intelligence: How general is it? (pp. 19-37). Mahwah, NJ: Lawrence Erlbaum Associates.

Steiger, J. H. \& Lind, J. C. (1980). Statistically-based tests for the number of common factors. Paper presented at the Annual Spring Meeting of the Psychometric Society, lowa City.

Thurstone, L. L. (1938). The perceptual factor. Psychometrika, 3, 1-17.

Thurstone, L. L. (1947). Multiple factor analysis. Chicago: University of Chicago.

Vickers, D., Butavicius, M., Lee, M. D., \& Medvedev, A. (2001). Human performance on visually presented Traveling Salesman Problems. Psychological Research, 65, 34-45.

Vickers, D., Mayo, T., Heitmann, M., Lee, M. D., \& Hughes, P. (2004). Intelligence and individual differences on three types of visually presented optimisation problems. Personality and Individual Differences, 36, 1059-1071.

Wechsler, D. (1997). Wechsler Adult Intelligence Scale (3rd ed.). San Antonio: The Psychological Corporation.

Woodcock, R. W. \& Johnson, M. B. (1989). Woodcock-Johnson psycho-educational batteryrevised. Allen, TX: DLM.

\section{Acknowledgments}

We thank Chloë Mount for collecting the data. This research was supported by a Faculty of Health Sciences Small Grant to the authors. 